\title{
Study of the Effect of Turbulence on Cooling Tower Wakes
}

\author{
Veronika Barraclough ${ }^{1 *}$, Pavel Šafařík ${ }^{2}$ \\ ${ }^{1}$ Aerospace Research Centre, Aerodynamics Department, Beranových 130, 19905 Prague, Czech Republic \\ ${ }^{2}$ Czech Technical University in Prague, Department of Fluid Dynamics and Thermodynamics, Technická 4, 16607 Prague, Czech Republic
}

\begin{abstract}
A subject of an experimental fluid mechanics task was measurement of flow around hyperboloid-shaped cooling tower. Experimental part was provided by means of $2 \mathrm{D}$ Particle Image Velocimetry (PIV). The contribution contains results from PIV measurements in a range of Reynolds numbers varying from 43000 up to 53000 . The tunnels used for the measurement were two different types: Low Speed Wind Tunnel of Eiffel type (LSWT) with low rate of turbulence intensity and Boundary Layer Wind Tunnel (BLWT) with high rate of turbulence intensity.
\end{abstract}

Keywords: mechanics of fluids, flow around a bluff body, Particle Image Velocimetry, cooling towers.

\section{Introduction}

In this paper, results from mechanics of fluids field will be presented. It is about subsonic flow past a bluff body of cylindrical cross section, one of the classical problems still being solved both experimentally and numerically.

Topic for this contribution originated from one of the often-investigated problems of civil engineering, wind loading on cooling towers. Cooling tower (and naturally its Model designed for wind tunnel research) is classified as the bluff body, more accurately as the circular cylinder with varying diameter. This problem had been investigated according to requests from an industrial partner. Detailed description and results of this experimental phase can be found in [1]. Thereafter, this civil engineering problem turned into mechanics of fluids problem, when structures around the Model began to be investigated.

The civil engineering part was provided as pressure distribution measurement by pressure sensors. Then Particle Image Velocimetry (PIV) system was involved and used for several types of measurements. The main purpose of this measurement was to get some information about vicinity of the objects on a quality of wakes.

To get some knowledge about the fluid flow around such object, one can gain some inspiration from classical literature. For instance, Zdravkovich in his book [2] illustrates the cooling towers problem can be remotely compared to a circular cylinder problem. To get an approximate picture of the flow, it is therefore possible to come out of the mean flow topology of the cylinder, as depicted in Pattenden et al. [3] for instance. A detailed and thoroughly processed summary of the structures around a hyperboloid-shaped object, with many references to the relevant papers and literature, was given by Heseltine [4].

Still talking about the flow around the bluff body, a great deal of attention in this area is paid to the top part of a finite object. Sumner wrote a review of existing knowledge of this problematic - either experimental research or numerical calculations [5]. There is also a recent study of Gonçalves et al. [6], based on (among other topics) measurements provided by 2D PIV in a water channel at Re $=43000$. 
Rostamy et. al. published a study of the wake behind in-ratio L/D varying cylinders at $R e=42000$ [7]. Park and Lee published a research of structures of cylinders embedded in atmospheric boundary layers [8].

The brief excursion to the world literature shows especially, that the experimental research for a hyperboloid-shaped model is still challenging. The main reason is that the flow is influenced by many features and is three-dimensional.

One of the decisive factors having impact on wake appearance and behaviour is the quality of incoming flow. The experimental research on cooling tower models can be provided in conditions close to reality, it means in wind tunnels with modelled atmospheric boundary layer, which also means with high level of turbulence level. These results are very specific - the whole Model is submerged in the boundary layer and all structures and separation line are influenced by this. For general view on the flow around such body, the wind tunnel with low turbulence level should be used.

Originally, the Model of the cooling tower was placed in the wind tunnel for simulating of atmospheric boundary layer. The measurements provided by PIV included average velocity fields behind the cooling tower.

This experimental part was later extended to measurement in the second type of the tunnel with low rate of turbulence. Experimental results from this part can be found in [9].

This paper presents results from both wind tunnels with an intention of emphasizing, how perceptible is the effect of turbulence on a feature of the wake of the model. This finding is relevant for the basic research. The cooling tower Model is geometrically complicated, the flow in the Boundary Layer Wind Tunnel is complicated. To get more general view on such 3D body of low L/D ratio would worth it simplifying either geometry of the Model or flow field or both.

This research, this PIV measurement around the flow also revealed a structure, which forms in the wake of the model, called an arch vortex. The whole problem later extended into the measurement of this structure. The geometrical and aerodynamical characteristics, which aren't part of this paper, can be found in [10], [11].

\section{Experimental setup}

\subsection{Wind tunnels, a model}

The subject of the experimental fluid mechanics task was the measurement of flow around hyperboloid-shaped cooling tower of a power plant. The experimental part was provided by means of 2D Particle Image Velocimetry (PIV) as the quickest tool for instant view at the problem. The tunnels used for the measurement were two different types: the Low Speed Wind Tunnel of Eiffel type (LSWT) and the Boundary Layer Wind Tunnel (BLWT).
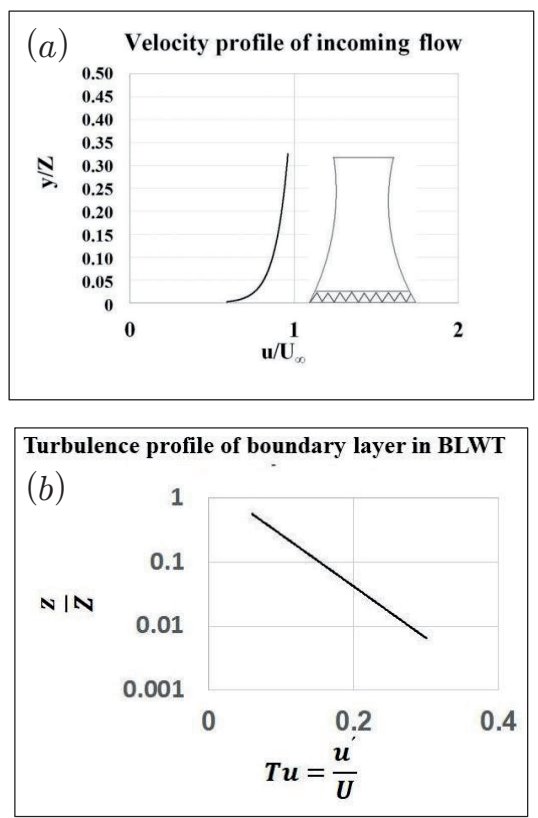

Fig. 1: (a) Characteristics of the flow in BLWT tunnel: Velocity profile of Incoming Flow. (b) Turbulence profile of boundary layer.

The physical modelling of this civil engineering problem sets the model into the BLWT, but because of the geometrical interest of the case, the Model was also measured in the LSWT with a low rate of turbulence intensity. There is a big difference in turbulence intensity between the two tunnels: while the LSWT has the turbulence intensity under $1 \%$, the turbulence intensity is intentionally a lot higher in the BLWT, as it is equipped with a special surface, which helps the development of the boundary layer. The model to thickness of BL rate reaches $1 / 3$ in a measurement area. Characteristics of the tunnel BLWT are depicted in Figs. 1 (a) and (b).

The LSWT wind tunnel test section has a diameter of 1.8 metres and the BLWT dimensions 
of the rectangular section are $1.8 \times 1.5$ metres. The measuring plane was set as parallel to the ground plane at an altitude of $0.2 \mathrm{~m}$ from the ground desk for both tunnels; in this height the model is narrowest.

The scale of the model was 1:400. The model cooling tower was printed on 3D printer as $0.32 \mathrm{~m}$ tall plastic object. The model was set on a board in the case of LSWT; short estimation of development of boundary layer on this board according to relation $\delta_{x} \approx 0.37 \cdot \sqrt{x} \cdot \operatorname{Re} e_{x}^{\frac{-1}{7}}$ set the thickness of the $\mathrm{BL}$ to $0.039 \mathrm{~m}$ at the position of the model. The model is depicted in Figs. 2 (a) and (b).
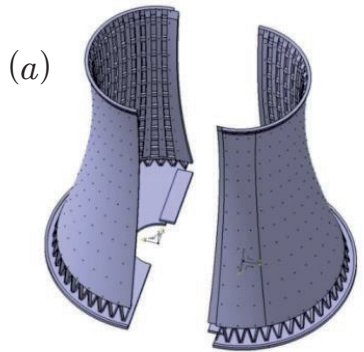

(b)

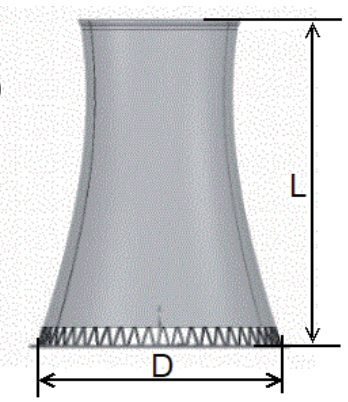

Fig. 2: (a) Characteristics of the flow in BLWT tunnel: Velocity profile of Incoming Flow. (b) Turbulence profile of boundary layer.

\subsection{Particle Image Velocimetry}

The experiment was provided in a classical way: the measurement area was seeded with olive oil droplets produced by Laskin nozzles and a green laser light was used to illuminate the area. The illuminated planes were captured by sensitive camera equipped by CMOS chip containing over $1 \mathrm{M}$ pixels, working in cross-correlation mode. The main 2D experiment was provided with a laser with Nd:YAG crystals and low frequency of the pulses. Figures 3 (a) and (b) depict the setup of the measurement, planes illuminated by laser light in the wake of the model. The maximum laser frequency was $15 \mathrm{~Hz}$, one measurement sequence consisted of 900 pictures. A software Dynamic Studio was used for capturing and evaluating results.

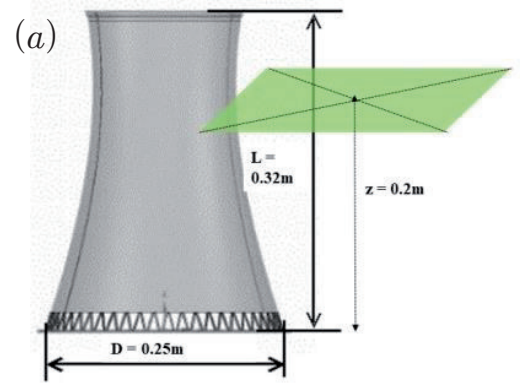

(b)

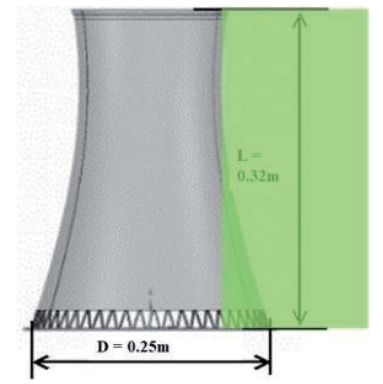

Fig. 2: (a) Characteristics of the flow in BLWT tunnel: Velocity profile of Incoming Flow. (b) Turbulence profile of boundary layer.

\section{Results}

3.1 Results from the tunnel LSWT with low rate of turbulence intensity

Figures 4 to 6 show an average velocity field behind the model in the wind tunnel LSWT with low rate of turbulence intensity. Figure 4 depicts the velocity in horizontal plane, 0.2 metres above the ground. Figure 5 depicts the velocity field in vertical cut (in the model axis), the bottom part of the model with recirculating area. Figure 6 depicts the same vertical plane, the top part of the model.

The Figure 7, vector line calculated from the averaged velocity field, reveal a possible structure forming in the wake, called the arch vortex. The figure shows the cut through its horizontally situated part. 3.2 Results from the tunnel BLWT with high rate of turbulence intensity

Figures 8 and 9 show an average velocity field behind the model in the wind tunnel BLWT with high rate of turbulence intensity. Figure 8 depicts the velocity in horizontal plane, 0.2 metres above the ground. Figure 9 depicts the velocity field in vertical cut (in the model axis). Figure 10 is similar to the Fig. 7: vector line calculated from the averaged velocity 

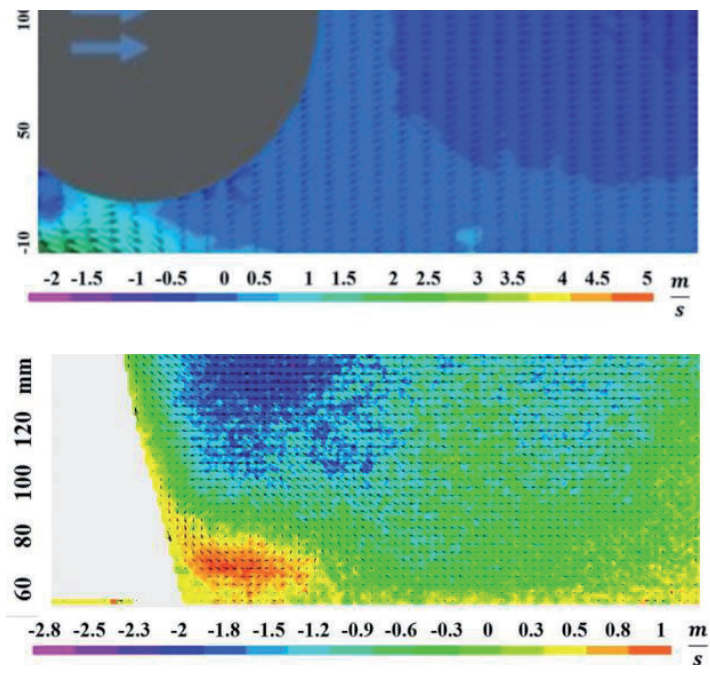

Fig. 4 (up): Averaged velocity field in the wake of the model in LSWT tunnel, showing the range of $x$-component of velocity from $-1 \mathrm{~m} / \mathrm{s}$ to $5 \mathrm{~m} / \mathrm{s}$. The depicted plane was situated parallel to the ground, at $z=0.2 \mathrm{~m}$ height.

Fig. 5 (down): Averaged velocity field in the wake of the model in LSWT tunnel, showing the range of $x$-component of velocity from $-3 \mathrm{~m} / \mathrm{s}$ to $1 \mathrm{~m} / \mathrm{s}$. This figure emphasizes the bottom part of the model.
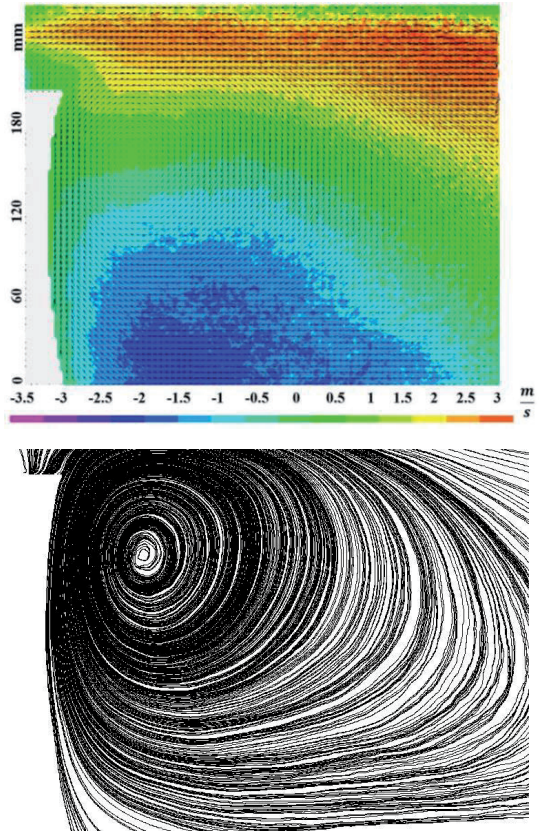

Fig. 6 (up): Averaged velocity field in the wake of the model in LSWT tunnel, showing the range of $x$-component of velocity from $-3 \mathrm{~m} / \mathrm{s}$ to $4 \mathrm{~m} / \mathrm{s}$.

This figure depicts the top part of the model. Fig. 7 (down): vector line calculated from the averaged velocity field.
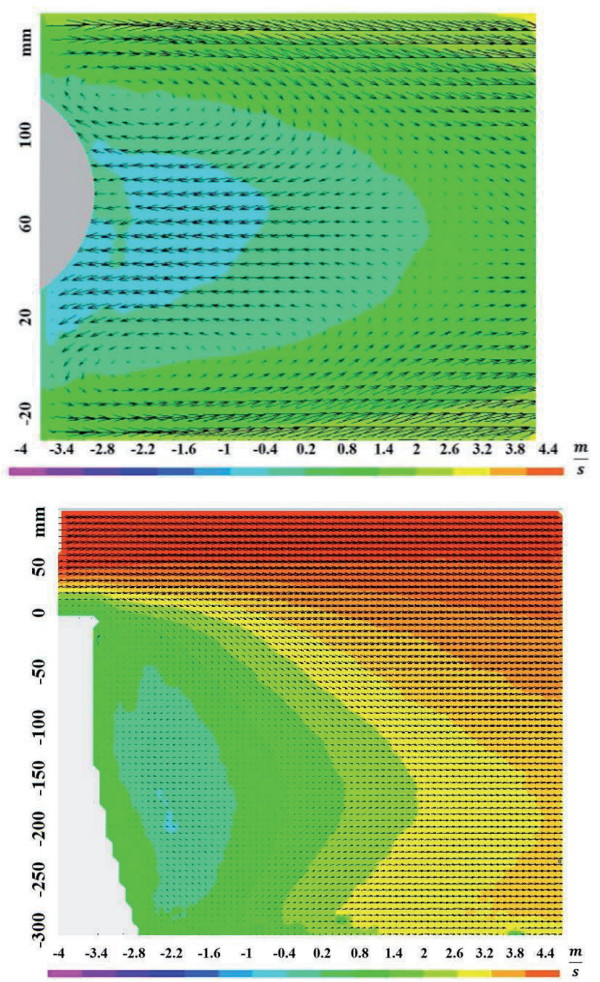

Fig. 8 (up): Averaged velocity field in the wake of the model in BLWT tunnel, showing the range of $x$-component of velocity from $-1 \mathrm{~m} / \mathrm{s}$ to $5 \mathrm{~m} / \mathrm{s}$. The depicted plane was situated parallel to the ground, at $z=0.2 \mathrm{~m}$ height.

Fig. 9 (down): Averaged velocity field in the wake of the model in BLWT tunnel, showing the range of $x$-component of velocity from $-3 \mathrm{~m} / \mathrm{s}$ to $1 \mathrm{~m} / \mathrm{s}$

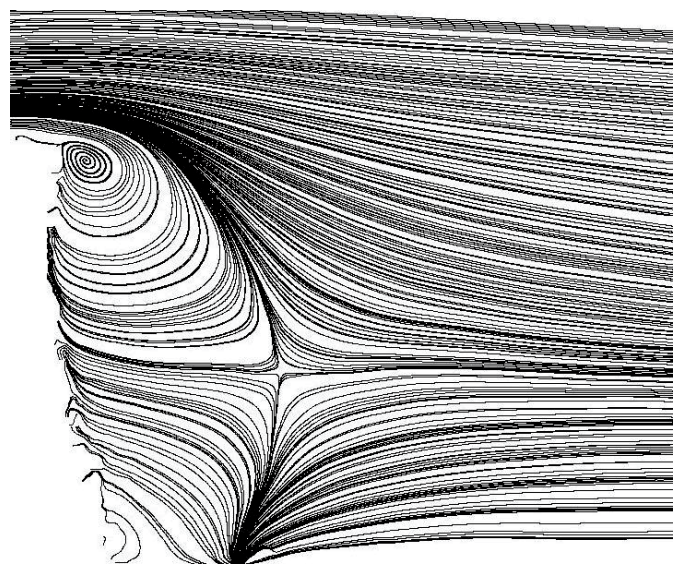

Fig. 10: Vector line calculated from the averaged velocity field, in the plane perpendicular to the ground. 
field, showing a recirculating area in the plane perpendicular to the ground. This picture shows also the position of the arch vortex. In comparison to Fig. 7, it is obvious, how the high rate of turbulence intensity of the incoming flow influences the wake structures for the same model.

The recirculating area for the wake in this type of tunnel is shorter, with the arch vortex top locked to the model. Similar results are revealed from the averaged velocity fields. Figure 6 and 9 compare the wake in the plane perpendicular to the ground. The numerical expression of this comparison is depicted in Fig. 11.

\section{Velocity profiles in the wakes}

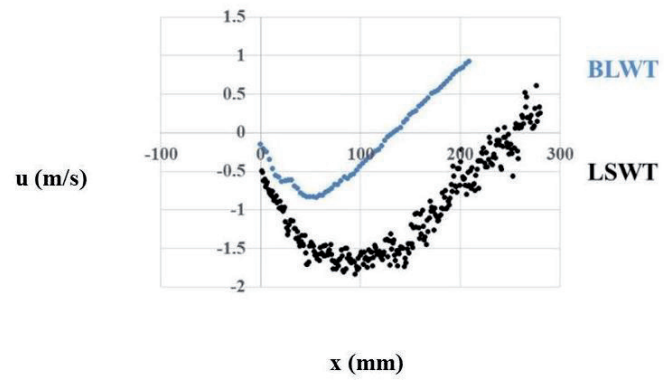

Fig. 11: X-components of the velocity profiles for the planes parallel to the ground. The $x$-axis beginning was set to the model wall.

\section{Conclusion}

The model of hyperboloid-shaped cooling tower was measured by means of Particle Image Velocimetry in two types of wind tunnels. One tunnel had the modelled turbulent boundary layer so the model was sunk in it, the second tunnel's turbulence intensity was under $1 \%$. The main intention of this contribution was to show the influence of turbulence intensity on the wake. The vector velocity fields in the planes parallel and perpendicular to the ground were measured and evaluated to show this phenomenon.

From the planes perpendicular to the ground, the $x$-component of velocity was calculated and clearly proved the difference between the wakes. The turbulent boundary layer supresses the wake and velocities in the $\mathrm{x}$-direction were as follows: the recirculating area for the wake in the BLWT is lot shorter and also the velocity $\mathrm{x}$-component is smaller.

\section{Acknowledgments \\ The research was performed with financial institutional support from the government budget through the Ministry of Industry and Trade of the Czech Republic.}

\section{References and Notes}

[1] Michálek, P., Zacho, D. (2014). Experimental investigation of group of cooling tower models in a wind tunnel (in Czech), internal report. Aerospace Research Centre, Plc, Prague, Czech Republic.

[2] Zdravkovich, M. M. (2003). Flow Around Circular Cylinders. Vol.2: Applications. Oxford University Press.

[3] Pattenden, R. J., Turnock, S. R., Zhang, X. (2005). Measurements of the Flow over a Low-Aspect-Ratio Cylinder Mounted on a Ground Plane, In: Experiments in Fluids 39, pp. 10-21.

[4] Heseltine, J. L. (2003). Flow around a Circular Cylinder with a Free End. A Thesis for the Degree of Master of Science, The Department of Mechanical Engineering, University of Saskatchewan, Canada.

[5] Sumner, D. (2013). Flow Above a Free End of a SurfaceMounted Finite Height Circular Cylinder: A Review. In: Journals of Fluids and Structures, vol. 43, pp. 41-63.

[6] Gonçalves, R. T., Franzini, G. R., Rosetti, G. F., Meneghini, J. R., Fujarra, A. L. C. (2015). Flow Around Circular Cylinders with Very Low Aspect Ratio. In: Journal of Fluids and Structures, vol. 54, pp. 122-141 (2015).

[7] Rostamy, N., Sumner, D., Bergstrom, D. J., Bugg, J. D. (2014). Local Flow Field of a Surface-Mounted Finite Circular. Cylinder, In: Journal of Fluids and Structures, vol. 34, pp. 105-122.

[8] Park, Ch.-W., Lee, S.-J. (2002). Flow Structure around a Finite Circular Cylinder Embedded in Various Atmospheric Boundary Layers. Fluid Dynamic Research, The Japan Society of Fluid Mechanics.

[9] Barraclough, V. (2015). A measurement of cooling towers models by means of PIV method (in Czech, internal report R-6180. Aerospace Research Centre, Plc., Prague, Czech Republic.

[10] Barraclough, V., Novotný, J., Šafařík, P. (2017). Experimental Investigation of 3D Vortex Structures in Wake of Hyperboloid-Shaped Model. Conference Topical Problems of Fluid Mechanics, Prague, Institute of Thermomechanics of Czech Academy of Science; Conference Proceedings.

[11] Barraclough, V. (2018) An Arch Vortex in a Wake of a Hyperboloid-Shaped Model, a doctoral thesis, Czech Technical University in Prague, Faculty of Engineering. 